\title{
Digitally printed photovoltaic devices with increasing stack complexity
}

\author{
Veronique Gevaerts, Anne Biezemans, Quentin Passet, Tamara Eggenhuisen, Robin Willems, Sjoerd \\ Veenstra, Jan Gilot, Jan Kroon, Ronn Andriessen
}

\author{
Solliance Solar Research, High Tech Campus 21, 5656 AE, Eindhoven, The Netherlands
}

\begin{abstract}
Digital printing of organic photovoltaic devices is presented as a viable option for the creation of increasingly complex device structures. Fully printed organic tandem junction devices were made that show a perfect summation of the open circuit voltages of the subcells. These results show the feasibility of complete digitally printed device stacks providing product designers with unprecedented freedom of design for integration of a photovoltaic functionality in new products.
\end{abstract}

\section{INTRODUCTION}

The development of solution processed solar cells is motivated by the low cost potential of the technology combined with the unique possibilities to integrate photovoltaic functionality in a large variety of applications. The low cost potential is based on the use of inexpensive, abundant materials and the high production speeds enabled by roll-to-roll (R2R) printing and coating. Although slot die coating is today the most applied R2R solution process method for solution processed organic photovoltaic devices, digital printing has recently shown to be an attractive method to deposit certain layers of a photovoltaic device stack.

The most efficient organic solar cells to date are using two different active layers in a series connected tandem device. Now these world record OPV devices have efficiencies in the double digits [1,2], using up-scalable technologies to produce these increasingly complex devices is needed.

\section{EXPERIMENTAL APPROACH}

Solution processing of OPVs on industrial scale brings some challenges, such as finding alternative, non-chlorinated solvents and using roll-to-roll compatible processes. Here we present a fully inkjet printed tandem OPV device, which consists of up to 9 sequentially inkjet printed layers as shown in Figure 1. An alternative structure using ITO and $\mathrm{MoO}_{3} / \mathrm{Ag}$ contacts was used in parallel. The inkjet printed layers are made without chlorinated solvents using an industrial scale printhead, making the processing industrial viable. One of the major challenges using inkjet printing is that the formation of the layers (wetting and pinning) depends not only on the solvent (mixture) used for the printing, but also on the surface energy of the layer/material the layer is processed on.

\section{MAIN RESULTS}

The main challenge for inkjet printing this tandem structure was to find a good way to print the recombination contact consisting of a closed PEDOT:PSS layer from a water based solution onto a hydrophobic P3HT:PCBM layer and on top of that a $\mathrm{ZnO}$ layer. Previously it was shown that $\mathrm{ZnO}$ can be inkjet printed on a PEDOT:PSS layer [3], however, when modifying (commercial) PEDOT:PSS formulations the resulting surface energy of this PEDOT:PSS layer can change and hence the printability of $\mathrm{ZnO}$ on top of the PEDOT:PSS is different. Careful optimization of the full recombination contact was done and tandem devices were successfully inkjet printed.

The voltage of the inkjet printed tandem we present here is the sum of the voltages of the subcells, which shows that there are no voltage losses over the recombination contact. The efficiency of the inkjet printed tandems was higher than the efficiencies of the respective single junction devices. These results show that inkjet printing is a promising technique to up-scale the production of tandem OPVs and commercialization is one step closer. a)

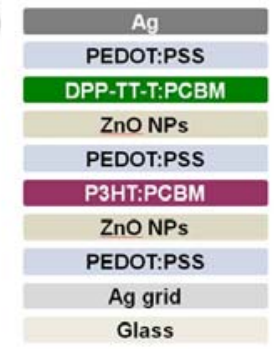

b)

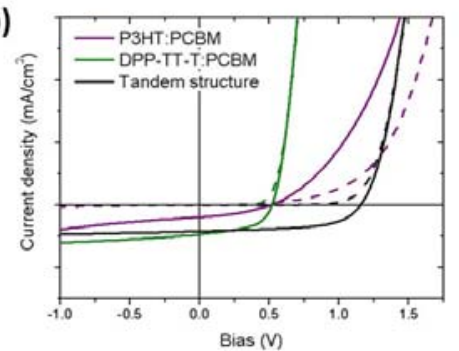

c)

\begin{tabular}{|l|l|l|l|l|}
\hline Device & $V_{\text {cc }}(\mathrm{mV})$ & $J_{\text {sc }}\left(\mathrm{mA} / \mathrm{cm}^{2}\right)$ & FF & Efficiency $(\%)$ \\
\hline Single junction P3HT:PCBM & 0.64 & 2.89 & 0.54 & 1.01 \\
\hline Single junction DPP-TT-T:PCBM & 0.54 & 5.55 & 0.51 & 1.52 \\
\hline Tandem junction & 1.18 & 4.65 & 0.60 & 3.27 \\
\hline
\end{tabular}

Fig. 1 a) layer stack of the all inkjet printed tandem device, b) $J-V$ curves of the respective single junction and tandem devices with ITO and $\mathrm{MoO}_{3} / \mathrm{Ag}$ contacts, c) solar cell characteristics of the best devices. 


\section{CONCLUSION AND DISCUSSION}

The results show that creation of multi junction devices using digitally mastered inkjet printing technology is possible when careful optimization of the sequential solvent systems is done. The inkjet printed layers are made without chlorinated solvents using an industrial scale printhead, making the processing industrial viable. Though the results are an important step forward in being able to process increasingly complex stack architecture using inkjet printing, the approach that was used in solvent engineering can also easily be transferred to slot-die coating or other large scale technologies.

\section{REFERENCES}

[1] G. Li, R. Zhu, Y. Yang, "Polymer Solar Cells", Nature Photonics, vol. 6, pp. 153-161, 2012.

[2] J. You, L. Dou, K. Yoshimura, T. Kato, K. Ohya, T. Moriarty, K. Emery, C.C. Chen, J. Gao, G. Li, Y. Yang, "A polymer tandem solar cell with $10.6 \%$ power conversion efficiency." Nature Communications, vol. 4, pp 1446, 2013.

[3] T.M. Eggenhuisen, Y. Galagan, E.W.C. Coenen, W.P. Voorthuijzen, M.W.L. Slaats, S.A. Kommeren, S. Shanmuganam, M.J.J. Coenen, R. Andriessen, W.A. Groen, "Digital fabrication of organic solar cells by inkjet printing using non-halogenated solvents”, Solar Energy Materials and Solar Cells, vol. 134, pp. 364-372, 2015 